Recibido: 22/07/2020

Aceptado: 30/07/2020

\title{
NEUROMARKETING E INNOVACIÓN
}

Neuromarketing and innovation

\author{
David Zambrano \\ dzambr@hotmail.com \\ Florida Global University
}

\section{RESUMEN}

En el presente estudio a través de una investigación documental, se describe el neuromarketing y su relación con la innovación, de cara a la evolución de los diferentes medios que se han puesto en práctica para obtener de forma cada vez más precisa una aproximación a la forma en que los consumidores perciben la realidad frente a determinados productos o servicios, de acuerdo a los estímulos que éstos puedan hacer llegar a través de los sentidos generando una determinada respuesta en cuanto a la decisión de compra frente a un producto o servicio respecto de otros. De allí que, haya sido necesario incorporar las herramientas que suministran las neurociencias para descubrir cuáles son estas percepciones $\mathrm{y}$, a partir de allí, definir las mejores estrategias de marketing para llegar hasta los clientes, captarlos y fidelizarlos. Todo ello conlleva a crear más interacción de las personas permitiendo que las empresas y las marcas sean percibidas más cercanas, propiciando una experiencia que los acerque cada vez más, lo cual exige una visión interdisciplinaria para poder estudiar y explicar los procesos clave del cerebro humano respecto a las decisiones de compra o adquisición al mismo tiempo, crear e implementar relaciones de largo plazo que conduzcan exitosamente a las organizaciones hacia sus metas teniendo como rutina una innovación constante.

Palabras clave: Neuromarketing, Innovación, consumidores.

\begin{abstract}
In the present study, through a documentary research, neuromarketing and its relationship with innovation are described, in the face of the evolution of the different means that have been put into practice to obtain an increasingly precise




\section{Revista científica, arbitrada e indizada, bajo la modalidad electrónica.}

approach to the way in which that consumers perceive reality in relation to certain products or services, according to the stimuli that they can send through the senses, generating a specific response regarding the purchase decision regarding a product or service compared to others. Hence, it has been necessary to incorporate the tools provided by neurosciences to discover what these perceptions are and, from there, define the best marketing strategies to reach, attract and retain customers. All this leads to creating more interaction between people, allowing companies and brands to be perceived closer, promoting an experience that brings them closer and closer, which requires an interdisciplinary vision to be able to study and explain the key processes of the human brain regarding to purchase or acquisition decisions at the same time, create and implement long-term relationships that successfully lead organizations towards their goals, routinely having constant innovation.

Key words: Neuromarketing, Innovation, customers.

\section{INTRODUCCIÓN}

Hoy en día, los clientes son cada vez más exigentes, lo cual aunado a la facilidad de acceso a un entorno global muy competitivo donde el mercado se ve influenciado por el fuerte desarrollo tecnológico, ha generado un aumento de la competencia a nivel universal, donde la relación con los clientes debe superar la simple colocación de un producto, bien o servicio, asegurando que regrese siempre atendiendo a sus niveles de satisfacción que serán la fuente de una relación que garantice futuros beneficios. En este sentido, en la actualidad alrededor del mundo se habla del Neuromarketing y su estudio de la mente humana donde se emplean instrumentos tecnológicos que permiten conocer al consumidor, sus emociones y cómo estas influyen en la decisión de compra. (Chipantiza, 2017)

A este respecto, los estudios de neuromarketing y el comportamiento del consumidor han sido mayores durante los últimos 10 años, puesto que el estudio del proceso de toma de decisión en los consumidores se ha convertido en un factor determinante para las empresas (Girón y Vásquez, 2018), en este sentido, 


\section{Revista científica, arbitrada e indizada, bajo la modalidad electrónica.}

el neuromarketing, se orienta al análisis del comportamiento del consumidor a través de la comprensión de sus procesos mentales para develar sus necesidades, sus deseos y emociones, sus sueños y aspiraciones para de este modo entender las razones por las cuales le hacen percibir, actuar $\mathrm{y}$ tomar decisiones de forma particular.

De igual forma, el neuromarketing facilita la identificación de los procesos racionales y emocionales los valores culturales, las creencias, las prácticas y la experiencia que afectan los sistemas neuronales subyacentes a una amplia gama de comportamientos humanos, desde la percepción y la cognición hasta la emoción, la cognición social y la toma de decisiones. (Chiao, 2009). Es por ello que, aporta a las empresas recursos de enorme valor para investigar el mercado, segmentarlo y desarrollar estrategias exitosas en posicionamiento de productos, precios, comunicaciones y canales de distribución para lo cual se integra la innovación constante aplicando estos descubrimientos y avances científicos.

De modo tal que, la innovación a través del neuromarketing, va a apoyar la detección de las necesidades básicas del consumidor, ocasionando una gran oportunidad en la sociedad, para que las nuevas empresas más allá de sus beneficios económicos y materiales, puedan innovar hacia un mejor mercado beneficioso que esté totalmente al alcance de la mayoría de los individuos.

\subsection{Del Marketing al Neuromarketing}

En el mundo globalizado empresarial actual, las organizaciones deben enfrentar continuamente distintas contingencias surgidas del entorno competitivo en el cual operan, de esta manera podrán mantenerse en el mismo, y crecer vertiginosamente, es decir, las organizaciones están condicionadas por un conjunto de factores de orden tanto endógeno y exógeno. Entre las de orden endógeno, se encuentran la tecnología de la empresa, su personal y la capacidad financiera, y en cuanto a las exógenas cabe señalar que el mercado es uno de 


\section{Revista científica, arbitrada e indizada, bajo la modalidad electrónica.}

los principales referentes que se toman en cuenta en una empresa para estimar y considerar las estrategias pertinentes que aseguren las ventas de sus productos y servicios.

A este respecto, el término marketing se suele asociar, o mejor confundir y con bastante frecuencia, con significados distintos; para muchos se corresponde con ventas, con publicidad, y/o investigación de mercados, para unos es un enfoque agresivo de mercado y para otros es una orientación dirigida al consumidor (Carasila y Milton, 2008), pero en todo caso se orienta a satisfacer las necesidades y expectativas del consumidor por lo que debe ir más allá de sólo estudiar la manera en que una persona compra productos tangibles también incluye el uso que hacen los consumidores de servicios, actividades, experiencias e ideas, por lo que ha requerido apoyarse en nuevos enfoques que le faciliten anticipar las potenciales decisiones de los consumidores.

En este sentido, el marketing tradicional comprende una disciplina que se basa en la investigación de los deseos e intereses de los consumidores para mejorar la comunicación a la hora de vender un producto o servicio. Los estudios son realizados, principalmente, a través de encuestas, entrevistas o estudios de comportamiento social (Luis y Rodríguez, 2016). Ahora bien, los cambios que se están produciendo en el marketing se deben al desarrollo de las nuevas tecnologías y con ello, su gran implicación en la vida de las personas y esto ha llevado a la creación de nuevos modelos de negocio para poder atraer a los consumidores de una manera diferente e innovadora. (Rodríguez 2015).

Por otra parte, el marketing estratégico comprende un análisis sistemático y permanente de las necesidades del mercado y el desarrollo de conceptos de productos rentables destinados a unos grupos de compradores específicos y que presentan cualidades distintivas que les diferencien de los competidores inmediatos (Lambin, 2003),de allí que la estrategia de marketing puede definirse como los diferentes planes o cursos de acción que puede elegir una empresa 


\section{Revista científica, arbitrada e indizada, bajo la modalidad electrónica.}

para reaccionar a las fuerzas ambientales que la afectan con tal de alcanzar los objetivos organizacionales dentro de un segmento de mercado (Mardones y Gárate, 2016). A este respecto, el concepto de estrategia de marketing se asocia a los elementos del marketing que determinen las ventas y beneficios de las empresas.

De este modo, surge el neuromarketing, que de acuerdo con Kothler citado por Brainon (2019), "es el uso de las neurociencias con la finalidad de facilitar y mejorar la creación, la comunicación y el intercambio de acciones, servicios y productos de valor entre grupos e individuos que necesitan y desean satisfacer sus necesidades mediante estos intercambios" (p. 1). En otras palabras se busca abordar el análisis del comportamiento del consumidor a través de la comprensión de sus procesos mentales, los cuales le hacen percibir, actuar y tomar decisiones de forma particular. Por tanto, la orientación principal de la Neurociencia para el neuromarketing radica en la comunicación a los clientes, especialmente al adentrarse en la mente humana, donde radica el subconsciente.

En consecuencia, el neuromarketing combina dos disciplinas: la neurociencia y el marketing, la primera tiene como objeto explicar la conducta en términos de actividades del encéfalo y cómo el medio ambiente $\mathrm{y}$ otros individuos influyen en ésta (Comins y París, 2013), en tanto que, el marketing se entiende, como una filosofía gerencial según la cual los objetivos organizacionales son alcanzados en la medida en que se identifiquen las necesidades y deseos de los consumidores o usuarios potenciales de los productos o servicios, y satisfacerlos a través de una oferta de valor superior a la competencia (Kothler y Armstrong, 2012). En otras palabras, aporta las implicaciones de la neurociencia al marketing para optimizar su campo de investigación.

En razón de lo anterior, se estudia el funcionamiento del cerebro, para saber su influencia en la decisión de compra, donde a través del estudio de estímulos en los sentidos se busca no perder de vista lo que genera el producto en el 


\section{Revista científica, arbitrada e indizada, bajo la modalidad electrónica.}

cerebro del consumidor, aplicando las técnicas de neuroimagen a la venta de productos o la captación de clientes potenciales (Victoria et al., 2015), por medio de esta herramienta cada organización espera alcanzar el éxito de su gestión efectuando importantes esfuerzos institucionales, destacándose que requieren contar con una estructura de marketing acorde a la naturaleza del negocio, además con la innovación tecnológica suficiente y necesaria que le brinde el soporte necesario.

En este orden de ideas, lo que se espera es comprender a través del apoyo de toda la tecnología disponible precisar el funcionamiento de los sistemas sensoriales del cerebro que codifican la información procedente del mundo exterior, es decir, cómo hace el sistema nervioso para traducir la enorme cantidad de estímulos a los que está expuesto un individuo al lenguaje del cerebro: activación y desactivación de neuronas, comunicación entre neuronas, transmisión de información y fenómenos que ocurren de cara a la exposición de los individuos ante la satisfacción de sus necesidades respecto a sus decisiones de compra.

Asimismo, Gómez y Niquepa (2013), explican que en un mundo tan competitivo y globalizado, la manera de conquistar al consumidor ha adquirido mayor importancia, hecho que se evidenció en el desarrollo de numerosas técnicas de investigación de mercados que pretenden entender las tendencias de consumo, con el fin de hacer más efectiva la comunicación entre cliente y productor que generen mayores beneficios económicos a los negocios. Por lo que, el objetivo principal de este nuevo campo es la aplicación de los hallazgos neurológicos de comportamiento de los consumidores utilizando métodos neurocientíficos que permitan anticipar comportamientos y decisiones. (Vásquez y Rueda, 2019) 


\section{Revista científica, arbitrada e indizada, bajo la modalidad electrónica.}

\subsection{Neuromarketing e Innovación}

El marketing y los negocios se han venido desarrollando a grandes escalas, a este respecto Klaric (2014), señala que la parte científica juega un papel muy importante para realizar procesos exitosos de innovación, este afirma lo siguiente: "En los 80 hablábamos un idioma funcional y servía, en el 2000 hablamos de emociones y también servía. Hoy si no trabajas con mensajes subconscientes instintos simbólicos, es muy difícil lograr la atención del consumidor" (p. 27). Ahora bien, teniendo en cuenta que el neuromarketing aporta de manera efectiva en los procesos publicitarios y de mercadeo, los profesionales del área, así como la gran mayoría de las empresas esperan lograr más creatividad y efectividad al momento de poder contar con herramientas innovadoras.

En este orden de ideas, orientado hacia la innovación el neuromarketing ha aportado diversas mediciones neurotecnológicas, mediciones biométricas y otras asociadas a respuestas eléctricas de la corteza cerebral como el Electroencefalograma (EEG) es una prueba que detecta la actividad eléctrica del cerebro mediante pequeños discos metálicos (electrodos) fijados sobre el cuero cabelludo. Las neuronas cerebrales se comunican a través de impulsos eléctricos y están activas todo el tiempo, incluso mientras duermes. Esta actividad se manifiesta como líneas onduladas en un registro de electroencefalograma.

Asimismo, efectúa otras mediciones como las imágenes por Resonancia Magnética Funcional (MRI), que representa la tecnología más avanzada en la actualidad para estudiar el cerebro, mide el componente de los glóbulos rojos (hemoglobina) encargado de transportar el oxígeno al resto de las células. Cada vez que se produce la sinapsis eléctrica, las neuronas extraen el oxígeno de las arterias, provocando un cambio en el campo magnético (Orus, Álava e Ibañez).

Además, la tomografía por emisión de positrones (PET), y, entre las más utilizadas, en mercadeo se pueden enunciar la respuesta electrodérmica, cuya 


\section{Revista científica, arbitrada e indizada, bajo la modalidad electrónica.}

medición permite inferir procesos atencionales y emocionales (Gutiérrez, 2019). De igual forma, se busca conocer la respuesta a la estimulación de los sentidos para intentar crear un ambiente agradable para el cliente y que, de este modo, se incremente el tiempo de permanencia en el establecimiento (Jiménez y Zambrano, 2017).

Asimismo, los movimientos oculares también denominada Eye Tracking, este sistema permite realizar un seguimiento de la mirada del individuo para identificar qué es lo que llama, capta o mantiene su atención ocular (Salamanca, 2018). De acuerdo con estas posibilidades de innovación tecnológica se hace necesario revisar en detalle el alcance de esta metodología ya que, según las zonas cerebrales que se activen, se podrá examinar entre muchos otros aspectos:

- Cuáles son los atributos de un producto o servicio que generan aceptación, rechazo o indiferencia.

- El nivel de aceptación (pre test) y recordación (pos test) de un comercial, en cualquiera de sus formatos: televisión, radio, gráfica, vía pública, etc., y el grado de impacto de cada una de sus partes, tanto en los aspectos neurosensoriales como en los relativos a los mecanismos de atención, emoción y memoria.

- La fuerza de los apegos emocionales a una marca en particular.

- Los estímulos que deben implementarse en un punto de ventas para incentivar las compras.

Por lo general, el inventario de los aspectos estudiados puede ser tan extenso como lo exija la gestión de marketing. En consecuencia, dependerá del nivel de importancia o profundidad con el cual la empresa enfoque su gestión, no sólo para desarrollar capacidades propias, sino también para lograr una mejor comprensión de los mecanismos que subyacen a todos los procesos de toma de decisiones de los clientes. Actualmente, la mayor parte de los estudios de este tipo se 


\section{Revista científica, arbitrada e indizada, bajo la modalidad electrónica.}

realizan en institutos especializados en los países más avanzados, ejemplo de éstos lo constituyen, entre otros Estados Unidos, Japón y Alemania.

En este sentido, se tienen las técnicas asociadas al método del reconocimiento facial. Al respecto, Peso-Viñals citado por Mantilla (2016), señala que: "una de las tecnologías más novedosas es una cámara HD capaz de identificar el movimiento de las facciones de una cara, que permiten identificar al momento lo que un usuario opina sobre un producto sin que entre en juego el pensamiento racional" (p.10). De acuerdo con el autor citado, a través de los micromúsculos del rostro de una persona, se puede capturar y definir la respuesta subconsciente de ese consumidor hacia un determinado artículo. De modo tal que, los movimientos de los músculos faciales reflejan el resultado directo del proceso subconsciente, la parte del cerebro que indica lo que realmente se piensa y se quiere.

En tal sentido, hoy en día se cuenta con software de procesos especializados, los cuales reciben los resultados brutos arrojados por cada tecnología implementada en un estudio de neuromarketing, que mediante diferentes cálculos algorítmicos, se obtiene un resultado específico que sirve para hacer una escala de efectividad e intención de compra que produce un estímulo determinado. Tomando en cuenta que el Neuromarketing es la manipulación del cerebro, a través de la exploración de los sentidos y los códigos que encajan en el ambiente social (Cisneros, 2012, p. 49). La anterior, es una excelente técnica para obtener información sobre el cliente respecto al producto, ya que analiza las primeras reacciones subconscientes e inconscientes, las cuales ayudan al consumidor para la toma de decisión de compra.

Ahora bien, las empresas que deseen implementar la innovación de una forma viable en medio de tantos productos y servicios que compiten por la atención y elección por parte del consumidor (Soto, 2014), solo queda por parte de éstas considerar utilizarla en forma integrada a las herramientas de 


\section{Revista científica, arbitrada e indizada, bajo la modalidad electrónica.}

neuromarketing, lo cual le permitirá desarrollar estrategias exitosas en materia de productos, diseño, marca, packaging, posicionamiento, precios, comunicaciones y canales, entre otros, soportadas mediante información altamente positiva para brindar una oferta diferenciadora y generadora de valor a sus clientes, mediante la implementación del neuromarketing, lo cual ofrece una mejora en la relación de las personas con la publicidad, los anuncios y el marketing en general. (Botello y Suárez, 2018).

\section{MATERIALES Y MÉTODOS}

El estudio se apoyó en una investigación de tipo documental de nivel descriptivo, ya que a través de ésta se ubicaron las fuentes necesarias vinculadas con la temática a desarrollar y se detallaron procesos y procedimientos necesarios en la aplicación del neuromarketing, apoyado además de la vinculación con la innovación respecto a las técnicas aplicadas para determinar las reacciones del consumidor.

En cuanto al método se utilizó el deductivo analítico, partiendo de una premisa general para obtener las conclusiones de un caso particular, sustentando en la teoría, la explicación y la abstracción. Para el acopio de las fuentes documentales, se empleó la revisión documental, el subrayado y el fichaje. Asimismo, se utilizaron como instrumentos los arqueos bibliográficos los cuales sirvieron a la vez de sistema de almacenamiento de la información procesada.

Para el análisis de las fuentes documentales, que permitieron abordar y desarrollar los requerimientos investigativos se emplearon el resumen analítico y el análisis crítico reflexivo. Estas técnicas implicaron, la lectura del material seleccionado, utilizando la técnica del subrayado de ideas principales y secundarias, para posteriormente, proceder a la realización de inferencias, razonamientos, comparaciones, argumentaciones, deducciones, críticas, estimaciones y explicaciones, entre otras. 


\section{Revista científica, arbitrada e indizada, bajo la modalidad electrónica.}

\section{RESULTADOS}

Se evidencia que una de las modalidades utilizadas son las neuroimágenes, que con el avance de las técnicas de neuroimagen no invasivas y el aumento del conocimiento sobre informática, matemáticas y cerebro humano produjo el mayor movimiento científico en torno a la comprensión de la estructura del cerebro humano y el sistema nervioso como nunca antes (Crespo y Legerén, 2018).

A través de estas, permite indagar qué está pasando en el cerebro de un consumidor ante los diferentes estímulos que recibe, lo cual brinda un campo de estudios mucho más amplio que el que ha ofrecido hasta ahora el marketing, por lo que sin duda, el creciente desarrollo de los equipos que exploran y, sobre todo, localizan las activaciones cerebrales ha dispuesto un amplio campo de estudios verdaderamente apasionante, con resultados que dejan atrás muchas limitantes con los respectivos supuestos previos, herramientas con las que el marketing tradicional no contaba, y que desde el aporte neurológico, es necesario considerar para tener interesado al consumidor. (Zuñiga y Cepeda, 2019).

Al respecto, Redolar (2014), argumenta que las neurociencias aplicadas al marketing intentan explicar el sistema nervioso desde la unidad estructural de menor tamaño que es la neurona, existiendo miles de millones que en conjunto desarrollan una comunicación química y eléctrica responsable de la conducta humana; dicha conducta estará determinada por las funciones mentales superiores (atención, aprendizaje-memoria, lenguaje, percepción, entre otras) y procesos emocionales que no solo son determinantes de conductas sino también de la interacción social del individuo y capacidad de decisión respecto a determinado producto.

De allí que, en cuanto a la compras se ha evidenciado que poseen una parte emocional concretamente se puede predecir el sesenta por ciento del comportamiento del consumidor a través de sus métricas emocionales (García y 


\section{Revista científica, arbitrada e indizada, bajo la modalidad electrónica.}

Pérez, 2020), y son estos estímulos emocionales los que no contemplan el marketing tradicional con tanta profundidad. En numerosas ocasiones estos estímulos escapan a nuestra razón $y$ se toman decisiones de compra inconsciente, por ejemplo, cuando se utiliza la resonancia magnética funcional por imágenes (MRI), cada exploración permite ver cómo y dónde se activa el cerebro ante cada estímulo mientras éste trabaja, al lograr comprender estos estímulos se logra utilizar el neuromarketing como una fantástica herramienta de venta.

En este orden de ideas, las reacciones que los individuos experimentan o conciben ante las marcas, los productos, los servicios, las empresas y demás objetos o sujetos, son de vital importancia para el marketing si el objetivo, como de hecho ha de ser, es que se deseen posicionar en un determinado mercado (Restrepo, 2017). A este respecto, es fundamental que las organizaciones apliquen el neuromarketing, ya que en el actual mundo globalizado permite el incremento del consumo de bienes y servicios, partiendo desde el contacto visual hasta llegar a influir en la actividad sensorial. (Avendaño, Paz y Rueda, 2015).

De este modo, el neuromarketing trae consigo un conjunto de recursos de enorme valor e innovación, debido a que si se comprende el funcionamiento del sistema nervioso y se profundiza en la forma en que percibe el consumidor, vale decir, su realidad, se podrán identificar más claramente sus necesidades y, en consecuencia, se podría visualizar con anticipación las posibles respuestas deseadas. (Gómez y Bandres, 2019).

A este respecto, las empresas requieren cada día ser más efectivas en sus estrategias de marketing aprovechando la innovación y la tecnología disponible para predecir las decisiones de compra e incorporarla a su estrategia que es el camino global que ésta transitará para alcanzar sus objetivos en términos de los mercados y los negocios. (Arango., Branch., Péres; 2008). 


\section{Revista científica, arbitrada e indizada, bajo la modalidad electrónica.}

En razón a lo anterior, la innovación tiene un papel importante para el tejido empresarial y el entorno tanto de los negocios como del neuromarketing, pues tal como lo afirman Helfat citado por Giraldo y Otero (2016), este concepto hace parte de las capacidades dinámicas de la empresa y ella le permite crear, extender o modificar su base de recursos intencionalmente (p.4), atendiendo a las perspectivas que ha logrado predecir en sus clientes. En este sentido, La innovación es considerada uno de los fenómenos más sobresalientes de la historia económica de la humanidad, ésta domina el panorama del paradigma de la productividad y de los componentes de la función de producción, facilitando y posibilitando el crecimiento socioeconómico (Quiroga y Hernández, 2014).

Ahora bien, esa intencionalidad de la empresa de ampliar su oferta mediante estos caminos queda circunscrita a las expectativas de quienes utilizarán en su propio beneficio esa innovación a través del neuromarketing intentando observar, escuchar y hacerse las preguntas correctas para saber lo que podría funcionar, pero nunca copiarlo exactamente (Ivars, 2018), es decir, obtener respuestas a través de los consumidores mediante las compras realizadas o reacciones respecto a servicios, para que cada vez sea más oportuno establecer los procesos y las acciones que les conduzcan a adquirir unos productos $\mathrm{u}$ otros.

\section{DISCUSIÓN DE RESULTADOS}

El marketing tradicional o como hasta ahora se había conocido, ha ido evolucionando para adaptarse al avance de los nuevos tiempos de la mano de la tecnología e innovación que ha marcado el desarrollo sostenido en los últimos años de diferentes enfoques y técnicas que han conllevado a la adaptación mediante herramientas de comunicación con los consumidores, sin embargo, éstas han ido quedando rezagadas por los cambios que van aconteciendo en el mercado y en la forma de acceder a este, por lo que se han llevado a cabo numerosas investigaciones para determinar nuevas estrategias. 


\section{Revista científica, arbitrada e indizada, bajo la modalidad electrónica.}

En este orden de ideas, con el apoyo de la neurociencia aparece el neuromarketing, el cual ha contribuido a que se disminuya el nivel de incertidumbre respecto a la colocación y posicionamiento de los bienes, productos o servicios. Esta innovación en el campo del mercadeo, facilita a las empresas ser más eficientes, eficaces y por tanto, efectivas al momento de lanzar nuevos productos al mercado, esto es posible mediante el estudio del consumidor, en relación con los estímulos internos del cerebro que son captados a través de los sentidos, los cuales son generados por los productos o servicios y la respuesta subconsciente e inconsciente que se produce hacia éste.

Por otra parte, las empresas podrán mejorar su producto a tiempo, para que sea más atractivo y logre tener mayor éxito, una vez sea lanzado al mercado, así como también propiciar la generación, mantenimiento y renovación de un portafolio que permita una diversificación dirigida en base a resultados obtenidos previamente, garantizando un óptimo mix de productos a los consumidores con alta probabilidad de mantener relaciones de largo plazo con los clientes basados en una aproximación a la realidad que perciben los potenciales compradores.

En definitiva, el Neuromarketing es una herramienta que ha impulsado la innovación tanto en diversas organizaciones para generar técnicas de marketing y comercialización a partir de los estudios mentales del cerebro de sus consumidores, que apoyadas con el desarrollo de las nuevas tecnologías han ido evolucionando y al mismo tiempo entrado a otras esferas del conocimiento, aprovechándolo como valor agregado al sostenimiento de las empresas.

\section{CONCLUSIONES}

El hombre a través del tiempo siempre ha buscado respuestas a los eventos que van sucediendo a su alrededor, en el mundo de los negocios esto no ha sido la excepción por lo que el mundo empresarial ha ido transformándose en la medida de que se han dinamizado los nuevos enfoques de gestión estratégica 


\section{Revista científica, arbitrada e indizada, bajo la modalidad electrónica.}

donde el marketing juega un rol fundamental al facilitar una serie de informaciones tendientes a conocer las necesidades, deseos y expectativas de la razón de ser de las compañías, sus clientes, sabiendo que éstos no reaccionan todos de igual forma, se han hecho esfuerzos para que las estrategias de marketing sean lo más aproximadas posible a la realidad particular de los consumidores.

En este orden de ideas, el marketing también ha ido ajustándose a las nuevas tendencias y enfoques de abordar el mercado, que aunado a la planificación estratégica adoptó en su momento una visión de marketing estratégico orientado a situaciones específicas atendiendo a objetivos empresariales previamente establecidos ajustando la mezcla de mercado, a saber, producto, precio, plaza y promoción, no obstante, aun así el abordaje del consumidor presentaba brechas en cuanto a la precisión de su comportamiento y respuesta ante el núcleo de las estrategias implementadas, vale decir, la compra, que en definitiva es el propósito de las mismas.

De allí que, teniendo paralelamente el desarrollo de la neurociencia y su aporte en cuanto al estudio de las reacciones causadas en el cerebro humano ante diferentes estímulos se hayan incorporado las innovaciones alcanzadas en este campo al área de marketing potenciando su alcance a través del neuromarketing lo cual ha ampliado su espectro de acción tanto en el abordaje del consumidor y su respuesta en el mercado de productos y servicios así como también en la formulación y desarrollo de estrategias dirigidas a sacar provecho en modo previo al comportamiento de los consumidores.

\section{REFERENCIAS BIBLIOGRAFICAS}

Arango, M; Branch, J., Pérez, G. (2008). Factores de Innovación en Marketing Estratégico - Un Caso Aplicado al Sector del Mueble Hogar en Itagüi (Antioquia). Recuperado en fecha 28-09-2020 de: https://www.scielo.org.co/pdf/dyna/v75n155/a03v75n155.pdf $+\& c d=2 \& h l=e u \&$ $\mathrm{ct}=\mathrm{clnk} \& \mathrm{gl}=\mathrm{es}$

Avendaño, W., Montes, L., Rueda, G. (2015). Estímulos auditivos en prácticas de neuromarketing. Caso: Centro Comercial Unicentro, Cúcuta, Colombia Recuperado en fecha 10-11-2020 de: http://www.scielo.org.co/pdf/cuadm/v31n53/v31n53a11.pdf 


\section{Revista científica, arbitrada e indizada, bajo la modalidad electrónica.}

Brainon, M. (2019). El Neuromarketing crea tendencia. Recuperado en fecha 28- 09-2020 de: https://martinbrainon.com/inicio/el-neuromarketing-crea- tendencia/

Botello, S., Suárez, K. (2018). Tendencias de Investigación en Neuromarketing. Cuadernos Latinoamericanos de Administración, vol. XIV, núm. 27, 2018. Universidad El Bosque. Recuperado en fecha 31-10-2020 de: https://www.redalyc.org/jatsRepo/4096/409658132013/html/index.html

Carasila, C., Miltón, A. (2008). El concepto de Marketing: pasado y presente. Revista de Ciencias Sociales v.14 n.2 Marcaibo ago. 2008. Recuperado en fecha 31-10-2020 de: http://ve.scielo.org/scielo.php?script=sci arttext\&pid=S131595182008000200014

Chiao, J. (2009). Neurociencia Cultural: Influencias Culturales en la Función Cerebral. Volumen 178. 1ra Edición. Madrid: Elsevier.

Chipantiza, E. (2017). El Neuromarketing en la Gestión Empresarial. Universidad Técnica de Ambato. Recuperado en fecha 05-11-2020 de: https://repositorio.uta.edu.ec/bitstream/123456789/26107/1/557\%20MKT\%20 sp.pdf

Cisneros, A. (2012). Neuromarketing y neuroeconomía: Código Emocional del Consumidor. 1ra. edición. México: Ecoe.

Comins, I., París, S. (2013). Los Desafíos de la Neurociencia. Un análisis desde la Filosofía para la Paz. Convergencia vol.20 no.62 Toluca may./ago. 2013. Recuperado en fecha 05-11-2020 de: http://www.scielo.org.mx/scielo.php?script=sci arttext\&pid=S140514352013000200004

Crespo, V., Legerén, B. (2018). El uso de la Neurociencia en el diseño de contenidos transmedia en los canales de televisión públicos de Europa: Videojuegos y Social TV. Edmetic. Recuperado en fecha 08-11-2020 de: https://dialnet.unirioja.es/descarga/articulo/6539975.pdf

García, R., Pérez, A. (2020). Comunicación y Educación en un Mundo Digital y Conectado. Icono 14. Indixado Scopus. Vol. 18, Núm. $2 \mid 2^{\circ}$ semestre (juliodiciembre). Recuperado en fecha 07-11-2020 de:

https://icono14.net/ojs/index.php/icono14

Giraldo, W., Otero, M. (2017). La Importancia de la Innovación en el Producto para Generar Posicionamiento en los Jóvenes. Rev.fac.cienc.econ., Vol. XXV (2), Diciembre 2017, 179-192. Recuperado en fecha 12-11-2020 de: http://www.scielo.org.co/pdf/rfce/v25n2/0121-6805-rfce-25-02-00179.pdf

Girón, B., Vásquez, K. (2018). Análisis de tendencias en Clústeres de investigación en neuromarketing y Negocios Internacionales. Universidad El Bosque, Bogotá. Recuperado en fecha 29-10-2020 de: https://repositorio.unbosque.edu.co/bitstream/handle/20.500.12495/3051/Gir $\% \mathrm{C} 3 \% \mathrm{~B} 3 \mathrm{n}$ Castellanos Betty Alejandra 2018.pdf?sequence $=1$ 


\section{Revista científica, arbitrada e indizada, bajo la modalidad electrónica.}

Gómez, M., Bandres, E. (2014). El neuromarketing: Una nueva disciplina para la investigación de audiencias y de la opinión pública. Icono 14. Indexado Scopus. Año 2014 Volumen $12 \mathrm{~N}^{\mathrm{o}} 2$ Recuperado en fecha 03-11-2020 de: https://icono14.net/ojs/index.php/icono14/article/view/666/498

Gómez, A., Niquepa, L. (2013). El Enfoque del Neuromarketing Aplicado al Mercado Colombiano. Universidad del Rosario. Recuperado en fecha 11-11-2020 de: https://core.ac.uk/download/pdf/86438751.pdf

Gutiérrez, G. (2019). El neuromarketing como herramienta efectiva para la educación en las ventas y la publicidad. Revista Latina de Comunicación Social, 74, pp. 1173 a 1189. Indexado Scopus. Recuperado en fecha 03-11- 2020 de: http://www.revistalatinacs.org/074paper/1377/60es.html

Ivars, A. (2018). La innovación en Marketing cuando (casi) todo está Inventado. https://es.semrush.com/blog/innovacion-en-marketing/. Recuperado en fecha: 1311-2020 de: https://es.semrush.com/blog/innovacion-en-marketing/

Jiménez, G., Zambrano, R. (2017). Marketing sensorial: merchandising a través de las emociones en el punto de venta. Análisis de un caso. Universitat Jaume I. Recuperado en fecha 09-11-2020 de:

http://repositori.uji.es/xmlui/handle/10234/173989

Klaric, J. (2014). Estamos ciegos. 3ra. Edición. Bogotá: Gestión 2000.

Kothler, P., Armstrong, G. (2012). Principios de Marketing. 10ma. Edición. Madrid: Pearson Prentice Hall.

Lambin, J. (2003). Marketing Estratégico. Tercera Edición. España: Editorial Mc Graw Hill.

Luis, C., Rodríguez, M. (2016). Neuromarketing, Un Nuevo Camino para Maximizar los Beneficios de tu Empresa. Recuperado en fecha 18-10-2020 de:https://riull.ull.es/xmlui/bitstream/handle/915/3561/Neuromarketing\%2C\%20u n $\% 20$ nuevo $\% 20$ camino $\% 20$ para $\% 20$ maximizar $\% 201$ os $\% 20$ beneficios $\% 20 \mathrm{de} \% 20 \mathrm{tu} \%$ 20empresa..pdf? sequence $=1 \&$ isAllowed $=\mathrm{y}$

Mantilla, L. (2016). Beneficios del Neuromarketing. Revista Difundiendo el conocimiento. Vol 2, No 1 (2016). Recuperado en fecha 30-09-2020 de: http://eduneuro.com/revista/index.php/revistaneuronum/article/view/21

Mardones, C., Gárate, C. (2016). Elementos de la estrategia de marketing y su efecto sobre la participación de mercado en la industria chilena. Universidad de Concepción. Contad. Adm vol.61 no.2 México abr./jun. 2016. Recuperado en fecha 01-112020de: $\quad$ http://www.scielo.org.mx/scielo.php?script=sci arttext\&pid=S018610422016000200243

Orus, C; Álava, P., Ibañez, S. (2018). Neuromarketing en Investigación de Mercados: una Revisión Práctica sobre su Pasado, Presente y Futuro. Recuperado en fecha: 12-11-2020 de: https://core.ac.uk/download/pdf/289997278.pdf 


\section{Revista científica, arbitrada e indizada, bajo la modalidad electrónica.}

Quiroga, D; Hernández, B; Torrent, J., Ramírez, J. (2014). La innovación de productos en las empresas. Caso empresa América Latina. Cuadernos del Cendes. Año 31. $\mathrm{N}^{\circ}$ 87. Tercera Época. Recuperado en fecha 11-11-2020 de: http://ve.scielo.org/pdf/cdc/v31n87/art04.pdf

Redolar, D. (2014). Neurociencia Cognitiva. España:Editorial Médica Panamericana.

Restrepo, S. (2017). Impacto del neuromarketing en la formación de profesionales en las áreas de publicidad, comunicación o marketing. Recuperado en fecha 2-11-2020 de:

https://www.researchgate.net/publication/327022655 NEUROMARKETING Y EM PRESA

Rodríguez, R. (2015). Marketing Experiencial: desde el Neuromarketing. Uva Segovia. Recuperado en fecha 14-10-2020 de: https://core.ac.uk/download/pdf/211097806.pdf

Salamanca, J. (2018). Las Redes Neuronales, la Creatividad, la Innovación y el Neuromarketing Presentes en la Gestión Administrativa de Hoy y del Futuro. Revista Unilibre No. 6 (2018). Recuperado en fecha: 05-10-2020 de: https://revistas.unilibre.edu.co/index.php/per ger humano/article/view/4500

Soto, C. (2014). E1 Neuromarketing Potencializa la Innovación de las Empresas. Revista eikon (1), 46-51. Recuperado en fecha 02-10-2020 de: https://app.eam.edu.co/ojs/index.php/eikon/article/download/98/123

Vásquez, L., Rueda, G. (2019). Tipos de Neuromarketing y herramientas para la medición de respuestas en el cerebro de los consumidores. III Congreso Internacional en Administración de Negocios Internacionales - CIANI 2019. Recuperado en fecha 05-11-2020 de: https://dialnet.unirioja.es/servlet/articulo?codigo=7274675

Victoria, J; Arjona, J., Repiso, R. (2015). El paradigma del neuromarketing a la Luz de su Producción Científica. Revista Venezolana de Información, Tecnología yConocimiento,ISSN 1690-7515,Vol.12, No.2, 2015, págs. 26-40. Recuperado en fecha:05-11-2020 de: https://dialnet.unirioja.es/servlet/articulo? codigo $=5178413$

Zuñiga, M., Zepeda, C. (2019). Uso de Herramientas del Neuromarketing: Un Estudio Empírico. IBIMA Science. Indixado Scopus. Recuperado en fecha 06-11-2020 de: https://ibima.org/accepted-paper/uso-de-herramientas-del-neuromarketing-unestudio-empirico/ 